\title{
Experience is a good, but not perfect, teacher
}

\author{
Stephen Balter
}

Received: 7 February 2009/Accepted: 2 March 2009/Published online: 15 March 2009

(C) Springer Science+Business Media, B.V. 2009

The paper "Determination of optimal viewing regions for X-ray coronary angiography based on a quantitative analysis of 3D reconstructed" models by Garcia et al. in this issue of the International Journal of Cardiovascular Imaging provides an instructive comparison between quantitative determination of beam angles and those routinely used in current clinical practice.

Optimum viewing angles were determined using segment by segment estimate of foreshortening and vessel overlap for each coronary artery segment in 137 patients. The data was then pooled to produce an optimum range of viewing angles for each segment.
The analysis largely confirmed conventional wisdom but did identify new and potentially useful projections for a few vessel segments.

The estimates of individual segment foreshortening provided in the present work could be of clinical use in situations where only 2D imaging is available. This data might improve the selection of device lengths.

The authors point out that optimum imaging angles for individual patients could be pre-computed when 3D data sets are available (e.g., CT angio). The use of individualized data should further improve angiographic and interventional practice.

Editorial comment to the article of Garcia et al. (doi: 10.1007/ s10554-008-9402-5).

S. Balter $(\square)$

Columbia University Medical Center, New York, NY,

USA

e-mail: sb2455@columbia.edu 